\title{
Bilateral surgical coronary ostial angioplasty with a superficial femoral artery patch in Takayasu aortitis
}

\author{
Hirokuni Arai, MD, Tetsuya Yoshida, MD, Satoru Hasegawa, MD, and Makoto Sunamori, MD, Tokyo, Japan
}

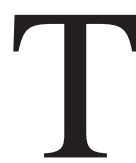

akayasu aortitis is a disease of unknown cause that affects mainly the aortic arch and the proximal portion of its main branches, including the coronary artery ostia. Narrowing of the coronary arteries is caused by intimal proliferation that extends into the arteries and contraction of the fibrotic media and adventitia that occurs in the aorta. Stenosis is therefore characteristically limited to the coronary ostia. Aorta-coronary bypass grafting and transaortic endarterectomy have been reported as treatments for this lethal condition. Late results have been unsatisfactory, however, because of the inflammatory disease process. ${ }^{1}$ We describe here successful bilateral surgical ostial angioplasty of the right coronary artery (RCA) and left coronary artery (LCA) in which superficial femoral artery patches were used in combination with aortic valve replacement in a patient with Takayasu aortitis.

From the Department of Cardiothoracic Surgery, Tokyo Medical and Dental University Graduate School of Medicine, Tokyo, Japan.

Received for publication March 13, 2002; accepted for publication April 18, 2002 .

Address for reprints: Hirokuni Arai, MD, Department of Cardiothoracic Surgery, Tokyo Medical and Dental University Graduate School of Medicine, 1-5-45 Yushima, Bunkyo-ku, Tokyo 113-8519, Japan (E-mail: hiro.tsrg@tmd.ac.jp).

J Thorac Cardiovasc Surg ;

Copyright $(\mathcal{C}$ by The American Association for Thoracic Surgery

$0022-5223 / \$ 35.00+0 \quad \mathbf{1 2 / 5 4 / 1 2 5 6 4 3}$

doi:10.1067/mtc.2002.125643

\section{Clinical Summary}

A 45-year-old woman had stable angina pectoris for several months. Coronary cineangiography revealed $90 \%$ stenosis of the LCA ostium and 50\% stenosis of the RCA ostium (Figure 1). The remainder of the coronary arteries appeared normal. Aortography revealed severe aortic regurgitation.

With the patient under neuroleptanalgesia and in the supine position, a median sternotomy was performed. A portion of the left superficial femoral artery (SFA) $6 \mathrm{~mm}$ in diameter and $8 \mathrm{~cm}$ in length was dissected and procured for an onlay patch. The open ends of the SFA were connected with an expanded polytetrafluoroethylene graft $6 \mathrm{~mm}$ in diameter. After extracorporeal circulation was begun, aortotomy was performed, and antegrade perfusion of crystalloid cardioplegic solution was achieved through each coronary ostium with a small, soft-tipped catheter.

For complete exposure of both the RCA and LCA takeoffs, the ascending aorta was completely transected about $10 \mathrm{~mm}$ above the sinotubular junction. The entire aortic wall showed severe, circumferential, fibrous thickening (4-5 $\mathrm{mm}$ in thickness) from the root to the mid level of the ascending aorta. Dense, fibrous tissue between the pulmonary artery and the aortic root was carefully resected, and the posterior wall of the sinus of Valsalva, including the left main trunk (LMT), was exposed by separating it from the main pulmonary trunk. Examination of the LMT from the lumen of the aorta revealed a stenotic orifice, narrowed by severe intimal thickening, that barely accepted a $1.25-\mathrm{mm}$ probe. The posterior aortic wall was pulled inferiorly with two pairs of 4-0 monofilament stay sutures. A V-shaped section of the aortic wall immediately above the LCA orifice was resected, beginning at the transverse aortotomy incision and continuing to the orifice. The aortotomy was
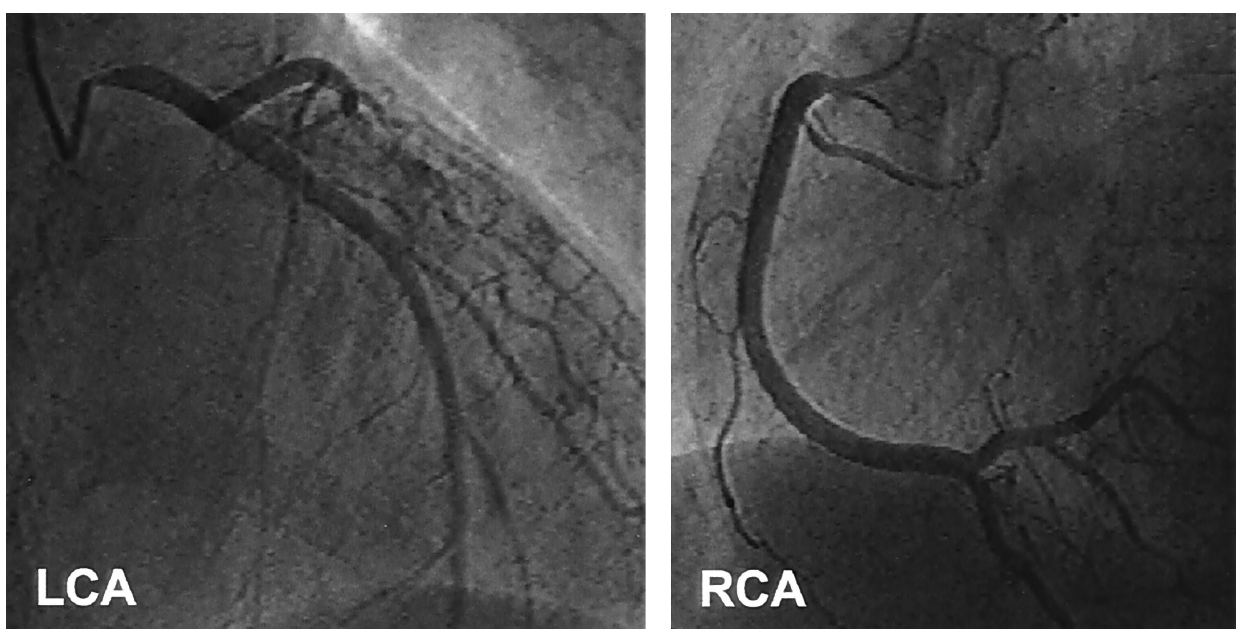

Figure 1. Preoperative coronary angiograms show stenoses of orifices of LCA $(90 \%)$ and RCA (50\%). 

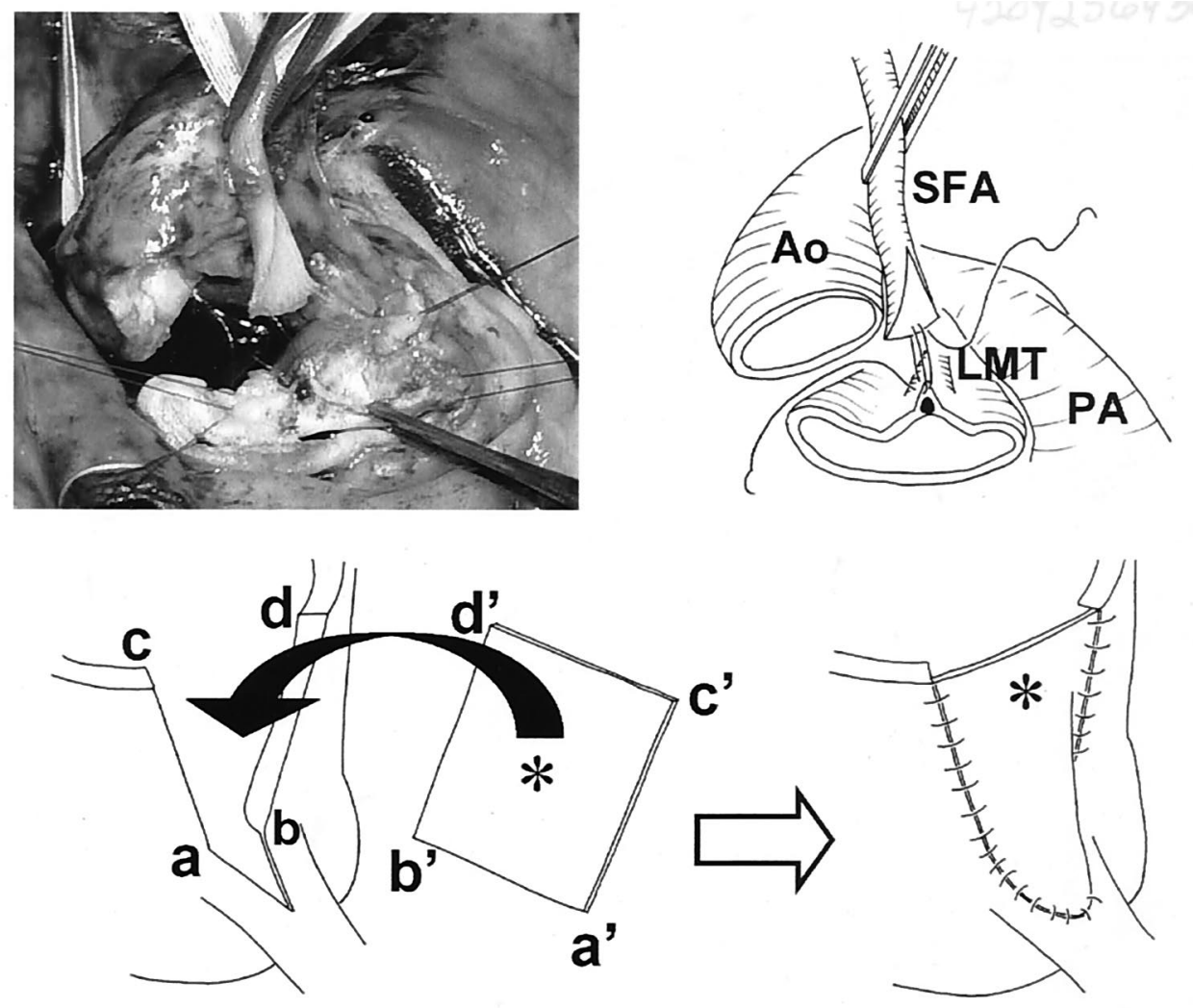

*: SFA patch
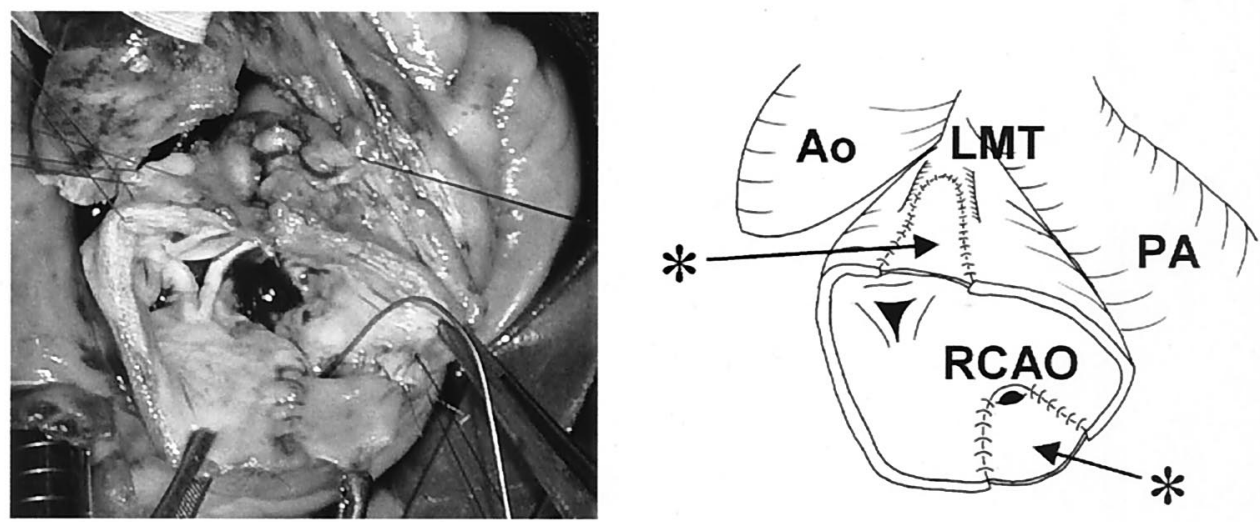

Figure 2. After complete transection of ascending aorta, V-shaped resection of aortic wall was made immediately above LMT orifice, and aortotomy was extended in straight line to roof of LMT. SFA patch was sutured with running monofilament suture (top). Rectangular SFA patch was sutured to create curtain fold that arched over roof of coronary artery takeoff, as indicated by matching of $a$ with $a^{\prime}, b$ with $b^{\prime}, c$ with $c^{\prime}$, and $d$ with $d^{\prime}$ (middle). Bilateral surgical coronary ostial angioplasty was completed with SFA patches, ensuring sufficient ostial orifice flow without kinking of patches (bottom). Ao, Aorta; PA, pulmonary artery; RCAO, RCA ostium.

extended to the roof of the LMT along a straight line about $9 \mathrm{~mm}$ in length. The intima of the LMT remained intact (Figure 2, top).

A rectangular patch was made by simple longitudinal incision of the resected SFA without additional trimming. The base of the patch was attached to the LMT with running 7-0 monofilament suture beginning at the inferior border of the LMT incision. Because the diameter of the SFA graft was about $6 \mathrm{~mm}$, the base of the rectangular patch was about $18 \mathrm{~mm}$ in length $(6 \pi)$, which was almost twice the length of the coronary incision. Accordingly, the lower two corners of the rectangular patch (corners $a^{\prime}$ and $b^{\prime}$, 

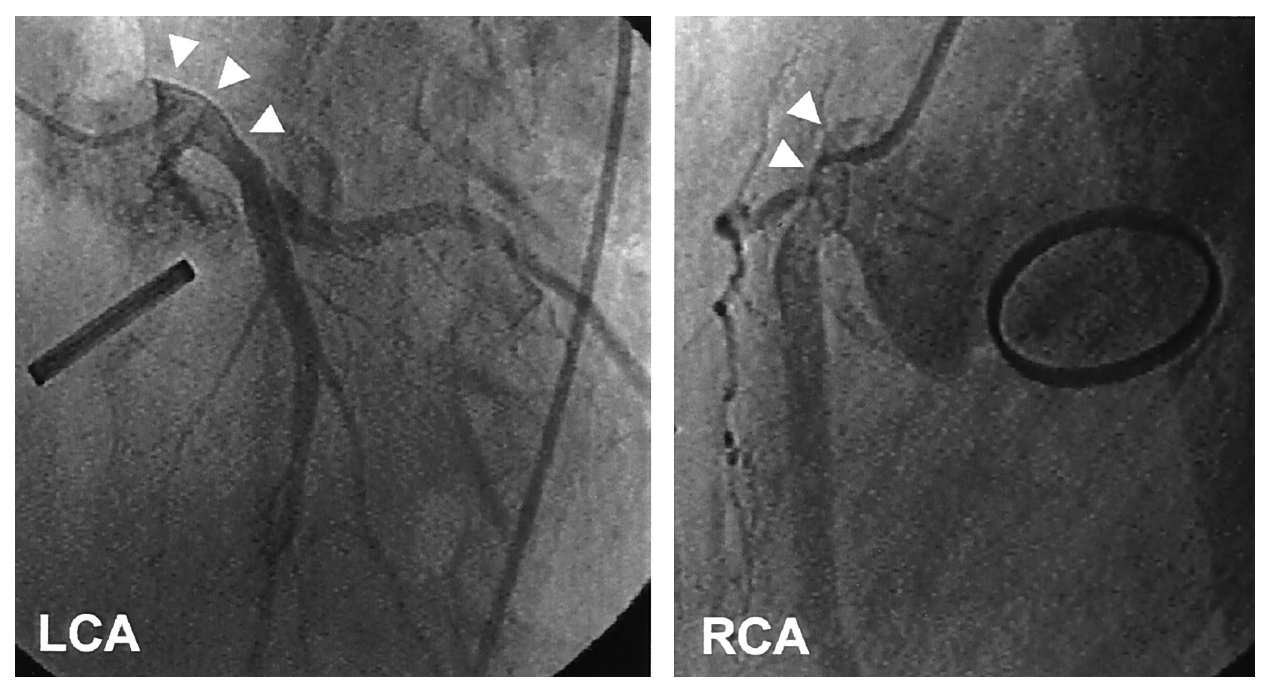

\section{Figure 3. Postoperative coronary angiograms show enlarged coronary ostia with excellent blood flow. Arrowheads indicate SFA patch.}

Figure 2, middle) corresponded to the junctions of the divided coronary artery ( $a$ and $b$, Figure 2, middle) with the aortic wall. Once the aortic wall was reached, the two longer sides of the rectangular patch were fitted to the $\mathrm{V}$-shaped aortic incision and were attached to the aorta with 6-0 monofilament suture. The patch was sutured in a manner that created a curtain fold that arched over the roof of the coronary artery, thus ensuring a sufficient orifice for the coronary ostium. We found it useful to leave a length of the femoral artery intact to facilitate handling of the arterial patch. After all suturing was complete, the patch was severed from the SFA graft at the level of the resected aorta.

The orifice of the RCA was also stenotic ( $2 \mathrm{~mm}$ in diameter), with severe intimal thickening. Another V-shaped aortic resection was performed, and a coronary arteriotomy was continued toward the roof of the RCA. The orifice of the RCA was constructed as described previously with a second patch, which was formed from the SFA graft left over after forming the LCA patch (Figure 2, bottom).

After construction of the coronary artery ostia, the aortic valve was removed and a St Jude Medical prosthesis (AT 23; St Jude Medical Inc, St Paul, Minn) was implanted with interrupted Ushaped mattress stitches of 2-0 Ethibond (Ethicon, Inc, Somerville, NJ) with polytetrafluoroethylene pledgets. The transected aorta was reconnected with continuous 4-0 monofilament suture. The patient was weaned from cardiopulmonary bypass without difficulty and transferred to the intensive care unit in good condition.

Histologic sections of the ascending aortic wall showed marked intimal proliferation, fibrous degeneration of the elastic fibers of the media, and some round cell infiltration of the adventitia. These findings were compatible with the chronic phase of Takayasu aortitis. Femoral artery histologic appearance was normal.

The patient was discharged in good condition without change in her electrocardiogram. Postoperative coronary angiography 1 month after surgery showed the sinus of Valsalva and the coronary ostia to be close in size and shape to the remodeling planned and then achieved at the time of surgery (Figure 3). It also showed no deformity at the roof of the LMT or RCA takeoff. At her 1-year follow-up, the patient reported no symptoms of ischemia during that first postoperative year.

\section{Discussion}

The most serious problem in coronary ostial plasty is kinking of the patch, which can lead to severe myocardial ischemia as a result of coronary flow disturbance. ${ }^{2,3} \mathrm{We}$ describe here a coronary ostial plasty technique in which a rectangular SFA patch is used. With the technique described here, we obtained excellent enlargement of both coronary ostia without kinking of the patches, thus ensuring sufficient bilateral coronary flow. In Takayasu aortitis, inflammation involving the SFA is uncommon. ${ }^{4}$ The SFA patches were easy to handle and easily attached to the thickened aortic wall. We believe the SFA patch to be better able to tolerate arterial pressure and thus to ensure graft function with less degenerative change in comparison to the autologous pericardial patch or saphenous vein graft, both of which can fail in the late term. However, there has not been enough experience with SFA patch grafts in the environment of the coronary ostium or sinus of Valsalva for the long-term results to be characterized. Thus it is mandatory to follow the clinical course and to evaluate the aortic root and the coronary ostia of each patient treated with this technique.

\section{References}

1. Amano J, Suzuki A. Coronary artery involvement in Takayasu's arteritis: collective review and guideline for surgical treatment. $J$ Thorac Cardiovasc Surg. 1991;102:554-60.

2. Sullivan JA, Murphy DA. Surgical repair of stenotic ostial lesions of the left main coronary artery. J Thorac Cardiovasc Surg. 1989;98:33-6.

3. Dion R, Verhelst R, Matta A, Rousseau M, Goenen M, Chalant C. Surgical angioplasty of the left main coronary artery. J Thorac Cardiovasc Surg. 1990;99:241-50.

4. Nakabayashi K, Nitadori T, Kamiya Y, Nagasawa T. Atypical Takayasu arteritis: late onset and arthritic manifestations: report of two cases. Int J Cardiol. 1998;66(Suppl 1):S221-7. 